\title{
Estomizado adulto no município de São Paulo: um estudo sobre o custo de equipamentos especializados
}

\author{
ADULT OSTOMY PATIENTS IN THE CITY OF SÃO PAULO: \\ A STUDY OF SPECIALIZED EQUIPMENT COSTS
}

\author{
ADULTO OSTOMIZADO DEL MUNICIPIO SAO PAULO: \\ UN ESTUDIO SOBRE EL COSTO DE LOS EQUIPAMIENTOS ESPECIALIZADOS
}
Vera Lúcia Conceição de Gouveia Santos ${ }^{1}$, Cristina Amoroso Damiani de Paula², Silvia Regina Secoli ${ }^{3}$

\section{RESUMO}

O estudo objetivou analisar o custo mensal do uso de dispositivos e adjuvantes por estomizados. Trata-se de uma pesquisa retrospectiva, realizada em dois Ambulatórios de Especialidades, em São Paulo. Os dados foram coletados em 635 prontuários de pacientes estomizados adultos, atendidos em junho de 2005. Os valores dos dispositivos e adjuvantes foram obtidos em registros eletrônicos e publicações oficiais da Secretaria de Estado da Saúde de São Paulo e seus resultados foram submetidos aos testes de Kolmogorov-Smirnov, MannWhitney, Kruskal-Wallis, Bonferroni e Spearman. A maioria dos indivíduos era do sexo feminino (51\%), idade $\geq 60$ anos, com colostomia provisória (64,5\%). 0 custo individual mensal médio foi $R \$ 137,72$, maior para os urostomizados, com estomas definitivos, com neoplasias de vias urinárias e atendidos no serviço que possui enfermeiro especialista. Houve correlação estatisticamente significativa e positiva entre o custo mensal e o tempo de estomia. Este estudo contribuiu para a avaliação do custo do estomizado no Estado de São Paulo.

\section{ABSTRACT}

This study was aimed at analyzing the monthly cost of the use of specialized equipment by ostomy patients. It is a retrospective study carried out at two Outpatient Health Centers in São Paulo. The data were collected in 635 records of adult ostomy patients assisted in June of 2005. For the costs, the data were obtained in electronic databases and official publications from the State of São Paulo Health Secretary, and the results were submitted to the Kolmogorov-Smirnov, MannWhitney, Kruskal-Wallis, Bonferroni and Spearman tests. Most of the individuals were female $(51 \%)$, aged $\geq 60$ years, with temporary colostomy (64.5\%). The average monthly cost was US\$ 51.0 per patient, higher for cancer urostomy patients with permanent stomas, with neoplasia in the urinary tract and assisted by the service with a therapist nurse. Statistically significant correlation between the monthly cost and the time with stoma was found. This study has contributed for the assessment of the cost of ostomy patients in the State of São Paulo.

\section{DESCRITORES}

Custos e análise de custo.

Equipamento/economia.

Colostomia/ economia.

lleostomia/economia.

Derivação urinária.

\section{KEY WORDS}

Costs and cost análysis.

Equipment/ economics.

Colostomy/economics. lleostomy/economics. Urinary diversion.

\section{RESUMEN}

En este estudio se tuvo como objetivo analizar el costo mensual del uso de dispositivos y adjuvantes por ostomizados. Se trata de una investigación retrospectiva, realizada en dos Consultorios externos de Especialidades, en Sao Paulo. Los datos fueron recolectados en 635 historias clínicas de pacientes adultos ostomizados, atendidos en junio del 2005. Los valores de los dispositivos y adjuvantes fueron obtenidos en registros electrónicos y publicaciones oficiales de la Secretaría de Estado de la Salud de Sao Paulo y sus resultados fueron sometidos a los tests de Kolmogorov-Smirnov, Mann-Whitney, Kruskal-Wallis, Bonferroni y Spearman. La mayoría de los individuos era del sexo femenino (51\%), edad $\geq 60$ años, con colostomía provisional $(64,5 \%)$. El costo individual mensual promedio fue de $\mathrm{R} \$$ 137,72 , mayor para los urostomizados, con estomas definitivos, con neoplasias de vías urinarias y atendidos en el servicio con enfermero especialista. Hubo correlación estadísticamente significativa y positiva entre el costo mensual y el tiempo de ostomía. Este estudio contribuyó para la evaluación del costo del ostomizado en el Estado de Sao Paulo.

\section{DESCRIPTORES}

Costos y análisis de costo.

Equipamiento/economía.

Colostomía/economía.

lleostomía/economía.

Derivación urinaria.

${ }^{1}$ Enfermeira especialista em estomaterapia. Professora Associada do Departamento de Enfermagem Médico-Cirúrgica da Escola de Enfermagem da USP. São Paulo, SP, Brasil. veras@usp.br 2 Enfermeira. Pós-graduada em estomaterapia pela EEUSP. São Paulo, SP, Brasil. cris_amoroso_hc@yahoo.com.br ${ }^{3}$ Enfermeira. Professora Doutora do Departamento de Enfermagem Médico-Cirúrgica da Escola de Enfermagem da USP. São Paulo, SP, Brasil. secolisi@usp.br 


\section{INTRODUÇÃO}

A necessidade e a problemática relacionada ao atendimento à pessoa com estoma é conhecida em todo o país. Atualmente, esse panorama é composto de alguns serviços de assistência, geralmente ligados a hospitais universitários, ambulatórios de especialidades, além de grupos de apoio e de associações, distribuídos de maneira desigual no território nacional e nem sempre providos de recursos materiais e humanos adequadamente qualificados. Nesse sentido, o restrito número de enfermeiros especialistas em estomaterapia - pouco mais de 300 - concentrados, principalmente, no estado de São Paulo, para uma população estimada em cerca de 170 mil estomizados no país ${ }^{(1)}$, confirmaria a deficiência nesse tipo de atenção.

Antes da inserção do estomizado na Coordenadoria Nacional para a Integração das Pessoas Portadoras de Deficiência (CORDE), normatizada pela Lei $n^{\circ} 7853$, em outubro de 1989, não havia critérios de planejamento para aquisição e distribuição de equipamentos coletores, adjuvantes e barreiras protetoras de pele, essenciais para o processo reabilitatório dessa clientela. Somente em 1993 e 1999, a Portaria no 116 (09/09/93), atualizada pela Portaria № 1230 (04/10/99), permitiram a inclusão de nove itens na tabela do Sistema de Informações Ambulatoriais do SUS (SIA - SUS). Apesar do alcance de ambas, mostraram-se limitadas não só por conterem especificações restritas e inadequadas, mas também, pela falta de uma política assistencial especializada(1-5).

Tendo em vista o aumento da produção de conhecimentos, na área da estomaterapia e da formação de estomaterapeutas, além do crescente desenvolvimento tecnológico dos equipamentos coletores, cada vez mais presentes no mercado nacional, tem-se delineado a necessidade de novos enfoques que gerem modelos assistenciais mais eficientes, pautados no atendimento interdisciplinar precoce, de caráter preventivo, individualizado e sistematizado, visando à reabilitação e à melhoria da qualidade de vida dessas pessoas ${ }^{(6)}$. Inclui-se nesse modelo, 0 enfoque farmacoeconômico, à luz da assistência especializada e da otimização dos recursos materiais, dentre os quais se incluem os equipamentos especificamente utilizados por esses usuários $^{(7-8)}$, que abrangem as bolsas coletoras (para estomas intestinais e urinários; para adultos e crianças); adjuvantes (desodorante, espessante, filtro, presilha, coletor urinário de perna e de cama, cateter urinário); de proteção e segurança (barreiras, cinto, removedor); para gastrostomias e sistemas de irrigação e oclusor de colostomia(a).

No cenário atual, a discussão referente ao uso da tecnologia na saúde, em particular os equipamentos cole-

(a) Consultar Política Nacional de Atenção às Pessoas com Estomas Disponível no site http://www.sobest.org.br tores, deve contemplar aspectos relacionados à avaliação dos custos, no sentido de garantir a viabilidade das instituições do sistema de saúde, assegurando a universalidade, eqüidade e qualidade no atendimento da população.

Considerando-se esses aspectos, os objetivos do presente estudo foram analisar o custo mensal do uso de equipamentos específicos por estomizados e verificar as associações existentes entre custo e as variáveis demográficas e clínicas.

\section{MÉTODO}

Trata-se de um estudo descritivo e retrospectivo, cuja realização foi precedida da aprovação do projeto pela Secretaria de Estado da Saúde de São Paulo (SES-SP).

Os dados foram coletados em dois ambulatórios de especialidades do município de São Paulo, ambos com atendimento de pessoas com estomas. 0 primeiro (A) abrange as regiões Leste, Oeste e Centro, atende cerca de 500 pacientes e 50 casos novos ao mês e conta com enfermeira pós-graduada em estomaterapia. 0 segundo (B), sediado em Santo Amaro, atende, em média, 200 pacientes e 20 casos novos mensalmente. Não possui especialista em estomaterapia em seu quadro de pessoal, embora conte com atendimento de enfermeira.

A casuística foi composta de 635 prontuários de todos os pacientes adultos (idade $\geq 17$ anos), com estoma intestinal e/ ou urinário, atendidos em junho de 2005 nos serviços citados. Assim sendo, a amostra ficou distribuída do seguinte modo: 486 indivíduos procedentes do Serviço A e 148 do Serviço B, correspondendo a $76,5 \%$ e $23,5 \%$ da amostra total, respectivamente.

Para a coleta de dados utilizou-se um instrumento composto de três partes: a primeira para obtenção das características demográficas; a segunda, para as características clínicas e a terceira, para identificação quantitativa e qualitativa dos equipamentos coletores, adjuvantes e protetores de pele distribuídos pelos serviços naquele mês de atendimento.

Obtidas essas informações, passou-se à estimativa dos custos dos equipamentos coletores, adjuvantes e protetores de pele identificados nos prontuários. Para a realização dessa etapa do estudo realizou-se consulta ao site www.pregao.gov.br, no qual foram acessados os Registros de Pregões negociados em 2004/2005, para obtenção dos respectivos números de Atas. Por meio desses, consultaram-se os Diários Oficiais do Estado de São Paulo, nos respectivos períodos de homologação, para acessar as Atas de Registro com os preços referentes aos produtos de compra, os códigos do Sistema Integrado de Informa- 
ções Físico-Financeiras (SIAFISICO) ${ }^{(b)}$ e os valores unitários dos produtos ${ }^{(c)}$.

Os valores dos equipamentos coletores, adjuvantes e protetores de pele foram registrados em uma planilha de materiais, composta dos itens relativos ao código (SIAFÍSICO) e ao valor monetário do produto (em reais).

Deve-se ressaltar que mais de 200 itens compõem os equipamentos coletores, adjuvantes e protetores de pele para estomizados, codificados no SIAFISICO da SES de São Paulo, e os serviços, credenciados junto ao Programa de Assistência ao Ostomizado da SES-SP, planejam e executam suas solicitações de compras de acordo com essa descrição, medida de suas demandas específicas.

Os dados foram armazenados e analisados no SPSS (Statistical Package for the Social Sciences), versão 13.0 SS. Os resultados foram submetidos aos testes de KolmogorovSmirnov, Mann-Whitney, Kruskal-Wallis, Bonferroni e Coeficiente de correlação Spearman. Valor de p inferior a 0,05 foi considerado para significância estatística $(p<0,05)$.

0 custo mensal dos pacientes foi do tipo direto e baseou-se apenas na categoria material, que incluiu equipamentos coletores, adjuvantes e protetores de pele utilizados pelo paciente durante o mês. Deste modo, o cálculo foi constituído pela somatória $(\Sigma)$ do valor unitário de cada item utilizado multiplicado pela freqüência de uso durante o mês, conforme a fórmula: $\mathbf{C}=\Sigma\left(\mathbf{C}_{1} \mathbf{x F} \mathbf{u}_{1}\right)+\left(\mathbf{C}_{2} \mathbf{x F} \mathbf{u}_{2}\right)+\left(\mathbf{C}_{\eta}+\right.$ $\mathbf{F u}$ ), onde $\mathrm{C}=$ Custo mensal individual; $\mathrm{C}_{1}=$ Custo unitário do item $1 ; \mathrm{C}_{2}=$ Custo unitário do item 2; $\mathrm{C}_{\mathrm{n}}=$ Custo unitário do item N; Fu =Freqüência de Uso no mês de cada item.

Nesse cálculo, não foram incorporados os custos indiretos, ou seja, aqueles resultantes do uso da infra-estrutura ambulatorial, do pagamento do pessoal envolvido na assistência e da perda de produtividade ou impacto na qualidade de vida do paciente.

\section{RESULTADOS}

Os achados do presente estudo mostraram que a amostra, em sua maioria, foi composta de indivíduos do sexo feminino (51\%), com média etária de 61,3+16,6 anos, com câncer colorretal (61,9\%, que possuíam colostomias (79,6\%), estomas provisórios $(64,5 \%)$ e operados há mais de 60 meses.

0 tempo médio de acompanhamento dos pacientes foi de 66,8 $\pm 28,5$ meses, com mediana 102,4 meses; superior para os usuários do Serviço $A$.

0 custo médio mensal por indivíduo foi $R \$ 137,70$, sendo significativamente maior no Serviço $A(R \$ 144,0)$.

Tabela 1 - Custo total de equipamentos coletores, adjuvantes e barreiras protetoras, segundo o Serviço - São Paulo 2005

\begin{tabular}{|c|c|c|c|c|}
\hline Variáveis (N) & Média (dp) & Mediana & Máximo-Mínimo & Kolmogorov-Smirnov (p-valor) \\
\hline \multicolumn{5}{|l|}{ Geral } \\
\hline Custo total (R\$) (634) & $137,7(97,8)$ & 112,6 & $890,4-13,4$ & $2,71(0,001)$ \\
\hline \multicolumn{5}{|l|}{ Serviço A } \\
\hline Custo total (R\$) (486) & $144,0(96,4)$ & 122,4 & $890,4-13,4$ & $2,49(0,001)$ \\
\hline \multicolumn{5}{|l|}{ Serviço B } \\
\hline Custo total (R\$) (148) & $117,2(99,8)$ & 87,2 & $638,8-13,7$ & $2,04(0,001)$ \\
\hline
\end{tabular}

Nota: $\mathrm{dp}=$ desvio padrão, $\mathrm{N}=$ número de indivíduos.

Tabela 2 - Custos médios mensais, segundo sexo e faixa etária, nos serviços estudados - São Paulo - 2005

\begin{tabular}{|c|c|c|c|c|c|c|c|c|c|}
\hline \multirow[t]{3}{*}{ Variáveis } & \multicolumn{9}{|c|}{ Unidades Estudadas } \\
\hline & \multicolumn{3}{|c|}{ Geral } & \multicolumn{3}{|c|}{ Serviço A } & \multicolumn{3}{|c|}{ Serviço B } \\
\hline & Média & dp & p-valor & Média & dp & p-valor & Média & dp & p-valor \\
\hline Sexo & & & $0,036^{\mathrm{a}, \mathrm{c}}$ & & & $0,089^{\mathrm{a}, \mathrm{d}}$ & & & $0,215^{\mathrm{a}, \mathrm{d}}$ \\
\hline Feminino & 146,5 & 104,8 & & 151,4 & 106,2 & & 129,3 & 98,6 & \\
\hline Masculino & 128,4 & 88,9 & & 135,8 & 83,8 & & 105,9 & 100,1 & \\
\hline Faixa Etária & & & $0,156^{\mathrm{b}, \mathrm{d}, \mathrm{e}}$ & & & $0,298^{\mathrm{b}, \mathrm{d}, \mathrm{e}}$ & & & $0,424^{\mathrm{b}, \mathrm{d}, \mathrm{e}}$ \\
\hline 17 a 25 anos & 96,8 & 65,0 & & 100,4 & 63,5 & & 92,0 & 71,6 & \\
\hline 26 a 59 anos & 135,2 & 95,2 & & 140,9 & 90,1 & & 124,1 & 104,0 & \\
\hline$\geq 60$ anos & 141,0 & 100,5 & & 146,7 & 100,2 & & 110,5 & 96,9 & \\
\hline
\end{tabular}

Nota: $d p=$ desvio-padrão, $a=$ Mann-Whitney $U, b=$ Kruskal-Wallis , $c=$ a diferença entre as médias é significativa ( $p<0,05)$, $d=a$ diferença entre as médias não é significativa $(p<0,05), e=$ Spearman

(b) Denominação técnica - específica das Secretarias de Saúde - usada para a descrição das características de materiais e equipamentos, disponíveis para distribuição aos usuários do SUS.

(c) http:// tabnet.datasus.gov.br/cgi/idb2004 [acessado em 15 de fevereiro de 2006]. 
Verificou-se diferença estatisticamente significativa $(p=0,036)$ entre os custos médios mensais para o sexo, sendo maiores entre as mulheres $(146,5 \pm 104,8)$. Não fo- ram constatadas associações estatisticamente significativas entre o custo mensal e idade, seja para a média ou para as diferentes faixas etárias.

Tabela 3 - Custos médios mensais segundo tipo e caráter do estoma, diagnóstico, tempo de estoma e de seguimento nos serviços - São Paulo - 2005

\begin{tabular}{|c|c|c|c|c|c|c|c|c|c|}
\hline \multirow[t]{3}{*}{ Variáveis } & \multicolumn{9}{|c|}{ Procedência } \\
\hline & \multicolumn{3}{|l|}{ Geral } & \multicolumn{3}{|c|}{ Serviço A } & \multicolumn{3}{|c|}{ Serviço B } \\
\hline & Média & dp & p-valor & Média & dp & p-valor & Média & dp & p-valor \\
\hline Tipo de estoma & & & $0,001^{b, c}$ & & & $0,001^{b, c}$ & & & $0,332^{b, d}$ \\
\hline Colostomia & 125,5 & 88,3 & & 128,7 & 83,0 & & 115,5 & 100,8 & \\
\hline Urostomia & 220,1 & 78,5 & & 222,4 & 77,3 & & 174,0 & 123,0 & \\
\hline Ileostomia & 170,1 & 127,1 & & 174,3 & 131,7 & & 139,0 & 83,3 & \\
\hline Caráter do estoma & & & $0,006^{\mathrm{a}, \mathrm{c}}$ & & & $0,115^{\mathrm{a}, \mathrm{d}}$ & & & $0,893^{\mathrm{a}, \mathrm{d}}$ \\
\hline Definitivo & 141,1 & 94,0 & & 145,1 & 94,5 & & 112,3 & 85,5 & \\
\hline Provisório & 131,0 & 104,4 & & 139,7 & 102,2 & & 119,6 & 106,6 & \\
\hline Diagnóstico & & & $0,001^{\mathrm{b}, \mathrm{c}}$ & & & $0,001^{\mathrm{b}, \mathrm{c}}$ & & & $0,354^{\mathrm{b}, \mathrm{d}}$ \\
\hline Câncer de cólon e reto & 131,2 & 92,3 & & 136,3 & 92,5 & & 111,8 & 89,6 & \\
\hline Câncer de bexiga & 220,0 & 97,8 & & 223,7 & 95,4 & & 147,5 & 160,5 & \\
\hline Trauma & 109,6 & 88,8 & & 115,9 & 82,7 & & 103,0 & 96,6 & \\
\hline Doenças inflamatórias & 49 & 7,8 & & 152,2 & 80,5 & & 201,3 & 173,1 & \\
\hline Outras & 143,9 & 91,4 & & 132,8 & 105,1 & & 112,3 & 90,1 & \\
\hline \multicolumn{10}{|l|}{ Tempo de estoma } \\
\hline$<1$ & 141,2 & 110,3 & $0,009^{\mathrm{b}, \mathrm{d}}$ & 175,8 & 116,1 & $0,066^{\mathrm{b}, \mathrm{d}}$ & 111,8 & 98,7 & $0,445^{\mathrm{b}, \mathrm{d}}$ \\
\hline $1 \vdash 3$ & 124,1 & 103,2 & & 170,6 & 127,3 & & 107,2 & 87,8 & \\
\hline $3 \vdash 6$ & 154,4 & 118,7 & & 156,7 & 104,7 & & 153,0 & 128,3 & \\
\hline $6 \vdash 12$ & 169,6 & 96,6 & & 171,7 & 96,7 & & - & - & \\
\hline $12 \vdash 36$ & 126,6 & 80,9 & & 129,7 & 81,2 & & 58,5 & 35,3 & \\
\hline $36 \vdash 60$ & 155,4 & 97,9 & & 153,8 & 95,5 & & 197,4 & 202,9 & \\
\hline$>60$ & 129,7 & 79,4 & & 130,9 & 79,9 & & 104,8 & 176,2 & \\
\hline \multicolumn{10}{|l|}{ Tempo médio de } \\
\hline & 66,84 & 28,53 & $0,001^{\mathrm{c}}$ & 83,13 & 111,83 & $0,001^{\mathrm{c}}$ & 13,71 & 14,05 & $0,001^{\mathrm{c}}$ \\
\hline
\end{tabular}

Nota: $d p=$ desvio-padrão, $a=$ Mann-Whitney $U, b=$ Kruskal-Wallis com o teste de correção de Bonferroni, $c=a$ diferença entre as médias é significativa $(p<0,05), d=$ a diferença entre as médias não é significativa $(p<0,05)$.

Os indivíduos com urostomia apresentaram custo médio mensal significativamente superior aos portadores de colostomia e ileostomia, o que não foi observado quando realizada a análise segundo o serviço.

Embora no geral, o custo tenha sido maior para os indivíduos com derivações de caráter definitivo, ao serem comparadas as médias dos grupos, não houve diferença estatisticamente significante entre elas.

Em relação ao diagnóstico, observou-se que os indivíduos portadores de câncer de bexiga apresentaram custo significativamente superior ao dos portadores das demais doenças. Ao ser realizada a análise de múltiplas comparações, todas as médias diferiram entre si.
0 custo mensal médio diferiu de modo significativo, também, entre os pacientes conforme o tempo de estoma, sendo maior para os indivíduos que tinham entre 6 e 12 meses. Entretanto, ao ser realizada a análise de múltiplas comparações, essa diferença não se manteve.

\section{DISCUSSÃO}

No Brasil, como ocorre em outros países, há um aumento de gastos com a atenção à saúde, porém com restrições orçamentárias cada vez maiores. Este crescimento exponencial está diretamente relacionado a uma serie de fatores como o emprego de novas tecnologias; 0 aumento da expectativa de vida da população; o incremento 
da demanda; a universalização do acesso à saúde; a escassez de mão de obra qualificada, que acarreta baixa produtividade; a má gestão das organizações devida à incapacidade administrativa dos profissionais de saúde; e a não implantação do sistema do controle de custos, entre outros ${ }^{(9)}$. Com isso, torna-se fundamental a adoção de métodos de aferição de custos para que os serviços e ações, oferecidos pelo SUS como pelas instituições privadas, sejam realizados com eficiência.

A assistência ao estomizado, entre outras ações de igual significância, inclui a seleção e indicação adequada de equipamentos coletores e adjuvantes específicos, em busca da reabilitação e melhoria da qualidade de vida dessa clientela. Para o estomizado, a bolsa coletora representa o fator patognomônico, assim como a insulina o é para o diabético, ao constituir a extensão de uma parte corporal perdida ${ }^{(10)}$.

Deste modo, desenvolvida como componente indispensável, no início do século passado, atualmente a bolsa coletora quase esbarra na perfeição. Ao apresentar-se em diferentes estilos, tamanhos, aberturas - com ou sem diversos tipos de barreiras protetoras de pele, plana ou convexa, aberta ou drenável, tamanho normal ou pe-diátrico ou neonatal, uma ou duas peças - tem demandado inúmeros adjuvantes e acessórios que proporcionam maior segurança e conforto ao usuário. Apesar de tal evolução, o uso dos equipamentos coletores para esto-mizados insere-se nos tópicos que ainda exigem mais evidências científicas, principalmente no que se refere a algumas de suas características como ser aberta ou fechada, ter ou não barreira protetora e qual tipo de barreira, ter ou não convexidade, tempo de uso em diferentes tipos de estomas entre outras ${ }^{(11)}$.

Além disso, embora conste como um dos dez direitos do estomizado, ela nem sempre é democrática, isto é, nem sempre é acessível a todos os que a demandam. Ao tratarse de material de elevado preço isolado, fabricado por grandes companhias multinacionais, ela acaba não se tornando disponível e acessível à clientela, principalmente nos países em desenvolvimento, os quais, geralmente, também não dispõem de políticas públicas específicas de atenção, abrangentes e inclusivas, assim como o Brasil.

Esta investigação, inédita em nosso meio, acerca do custo mensal do uso de equipamentos específicos para estomizados em São Paulo, buscou subsidiar principalmente os estudos de impacto econômico, em andamento no M inistério da Saúde (à época de sua realização), no processo de avaliação da Política Nacional de Saúde das Pessoas com Estomas, recentemente elaborada pela Associação Brasileira de Estomaterapia (SOBEST) e Associação Brasileira de Ostomizados (ABRASO) (d).

(d) A política Nacional de Atenção às Pessoas com Estomias foi aprovada no Conselho Nacional de Saúde, em reunião de 13 de fevereiro de 2007 porém ainda não foi implementada.
Detectando-se um custo médio mensal inferior a US\$ 80.70 por paciente parece romper-se o mito do alto custo direto desse usuário ao SUS, mesmo considerando-se os locais de realização do estudo - dois serviços de referência vinculados à SES-SP. Todavia, há que se considerar que esses equipamentos são fornecidos pelo Estado, cujo custo mensal, estimado para essa amostra, seria de US\$50,543.06 e o custo anual de US\$ 606,516.76 - montante que, de fato, apresenta repercussão importante no orçamento destinado à saúde. Além disso, é importante lembrar que o presente estudo investigou uma casuística circunscrita ao município de São Paulo que, a despeito de concentrar o maior número de estomizados cadastrados no estado de São Paulo, não representa o total de atendimentos, o que, seguramente, ampliaria esse custo.

Embora incluindo somente 635 pacientes, atendidos durante um único mês, a amostra pode ser considerada representativa ao inserir um dos pioneiros e maiores centros de referência no atendimento de estomizados do país, além de período típico e não sazonal, em que não houve perdas de pacientes por falta de equipamentos.

Dentre os resultados obtidos, destacamse os custos mais elevados entre as mulheres, pacientes com câncer de bexiga e, conseqüentemente, urostomizados, e com maiores tempos de estoma e de seguimento no Serviço $A$, isto é, no qual há enfermeira especialista em estomaterapia.

Em nosso meio, o sexo feminino tem predominado, como característica demográfica, em diversos estudos realizados com pacientes ambulatoriais, embora não existam diferenças significativas entre os sexos na epidemiologia do câncer colorretal, ainda a causa mais freqüente dos estomas. No que se refere aos custos dos equipamentos, superiores entre as mulheres, pode-se considerar o grande impacto do estoma sobre a imagem corporal, vestuário e sexualidade o que poderia levá-las a um consumo mais elevado - seja dos equipamentos de uso diário seja de dispositivos adjuvantes, como cintos e barreiras protetoras, ou mais avançados, principalmente os oclusores de colostomia - visando à maior segurança e conforto, à preservação da aparência e da higiene bem como ao retorno precoce às atividades principalmente domésticas. Todas essas condições são essenciais à reabilitação. Outro fator causal do maior custo pode estar relacionado à presença de complicações, como dermatites, hérnias paracolostômicas, prolapsos e episódios diarréicos, bastante freqüentes em nosso meio ${ }^{(12-13)}$.

Embora se afirme que o envelhecimento populacional e 0 aumento da longevidade implicam em custos maiores relacionados a internação, tratamento, equipamentos e medicamentos mais dispendiosos devidos, principalmen- 
te, às maiores incidências de doenças crônico-degenerativas, nossos achados não mostraram associações significativas entre custos e idade mais avançada.

Para o tipo de estoma, verificou-se que, de maneira geral, os urostomizados - predominantemente por câncer de bexiga - gastam mais em equipamentos específicos comparativamente aos colostomizados e ileostomizados. Embora numericamente sejam, cada vez, em menor número, certamente as características do efluente e a presença de estenose e infecções urinárias, como complicações freqüentes, acarretam o maior consumo. Além disso, esses equipamentos são proporcionalmente mais caros não só pela compra em quantidades menores como, principalmente, pelas suas características mais complexas ao exigirem plásticos mais resistentes ao odor e barreiras de maiores aderência e durabilidade e, conseqüentemente, maior segurança(14).

Resultados interessantes, aparentemente controversos, dizem respeito aos maiores custos de utilização de equipamentos específicos entre pacientes com maior tempo de estomia e atendidos em serviço especializado, isto é, com estomaterapeuta. Parece-nos que o maior desenvolvimento de habilidades no manejo do estoma, pele e equipamento - adquirido ao longo do tempo - e as orientações sobre 0 auto cuidado - oriundas de seguimento especializado - tenderiam a reduzir os custos. Por outro lado, poder-se-ia considerar que tais condições favoreceriam 0 aumento de demandas por procedimentos e equipamentos de maior complexidade e melhor qualidade bem como com diferentes características para ocasiões distintas, exatamente à medida da re-significação da própria identidade, da maior autonomia na realização do auto-cuidado, auto conhecimento, auto-estima além da gradativa reinserção nas atividades prévias à doença e à operação, bem como em novas atividades, em direção ao sucesso reabilitatório(10). Há que se considerar ainda que o Serviço $B$, além de não apresentar estomaterapeuta, tem deficiência de espaço físico, o que limita as atividades educativas ao estomizado quanto a alguns procedimentos mais complexos como o auto-cateterismo urinário, a irrigação e sistema oclusor de colostomia.

\section{REFERÊNCIAS}

1. Santos VLCG. Cuidando do estomizado: análise da trajetória no ensino, pesquisa e extensão [tese livredocência]. São Paulo: Escola de Enfermagem, Universidade de São Paulo; 2006.

2. Carvalheira C. A realidade das associações de estomizados no país. In: Santos VLCG, Cesaretti IUR. Assistência em estomaterapia: cuidando do ostomizado. São Paulo: Atheneu; 2000. p. 303-15.
Em revisão bibliográfica, nos últimos cinco anos, encontraram-se somente publicações relacionadas à apresentação e teste de novos produtos ${ }^{(15-18)}$ e à comparação das barreiras protetoras de óxido de zinco e hidrocolóide e suas ações sobre a pele saudável|(19), além de atualização sobre os produtos disponíveis no mercado internacional ${ }^{(20)}$. Embora esses estudos contribuam para a melhoria das evidências acerca da indicação e utilização dos equipamentos coletores, adjuvantes e protetores de pele para estomizados, não incluíram avaliação de custos. Além disso, são todos estudos internacionais, não sendo encontrada qualquer publicação nacional que desse suporte aos nossos achados.

\section{CONSIDERAÇÕES FINAIS}

Este estudo permitiu estabelecer o custo mensal do uso de equipamentos coletores, adjuvantes e barreiras protetoras de pele por estomizados, na cidade de São Paulo, mostrando-se relativamente baixo quando analisado isoladamente. Tais achados, mesmo que restritos, subsidiarão o M inistério e as Secretarias Estaduais e Municipais de Saúde na implemenmtação da Política Nacional de Atenção às Pessoas com Estomias, recém aprovada no país.

Por outro lado, o estudo também demonstrou existirem correlações do custo com importantes fatores como o sexo, diagnóstico, tipo de estoma e tempo de estomizado além de tempo de seguimento no serviço especializado, 0 que poderá colaborar para a melhoria do atendimento de demandas específicas, ao serem consideradas.

Apesar dos limites de sua casuística e de sua abrangência - com vistas a estimativas nacionais - certamente ele contribui como ponto de partida para o desenvolvimento de investigações mais amplas, que incorporem a farmacoeconomia como modelo teórico fundamental para estudos dessa natureza, não só na especialidade como na Enfermagem como um todo. A inclusão metodológica dos custos indiretos, em futuros estudos, aprofundará 0 impacto dos custose, conseqüentemente, do uso de equipamentos coletores também na esfera psicossocial dessas pessoas.

3. Santos VLCG. A estomaterapia através dos tempos. In: Santos VLCG, Cesaretti IUR. Assistência em estoma-terapia: cuidando do ostomizado. São Paulo: Atheneu; 2000. p. 1-17.

4. Brasil. Ministério da Saúde. Portaria n. 116, de 09 de setembro de 1993. Inclui no Sistema de Informações Ambulatoriais do SUS a concessão de bolsas de colostomia, garantindo 0 direito à cidadania plena através do fornecimento de equipamentos necessários para a promoção, prevenção, assistência e reabilitação da saúde dos ostomizados [legislação na Internet]. Brasília; 1993. [citado 2007 jan. 11]. Disponível em: http:/www.abraso.org.br 
5. Brasil. M inistério da Saúde Portaria n. 146, de 14 de outubro de 1993. Estabelece as diretrizes gerais para a concessão de próteses e órteses através da Assistência Ambulatorial, atribuindo aos gestores estadual/municipal de saúde as responsabilidades pela coordenação, supervisão, controle, avaliação e aquisição das próteses e órteses [legislação na Internet]. Brasília; 1993. [citado 2007 jan. 11]. Disponível em: http:// www.abraso.org.br.

6. Paula MAB, Santos VLCG. O significado de ser especialista para o enfermeiro estomaterapeuta. Rev Lat Am Enferm. 2003;11(4):474-82.

7. Bootman JL, Townsend RJ, McGhan WF. Principles of pharmaeconomics. $2^{\text {nd }}$ ed. Cincinnatti: Harvey Whitney; 1996.

8. Velasquez G. Farmacoeconomia: evolución científica a estrategia comercial. Pan Am J Publ Health. 1999;5(1):54-7.

9. Secoli SR. Farmacoeconomia da terapia analgésica utilizada na dor pós-operatória [tese]. São Paulo: Escola de Enfermagem, Universidade de São Paulo; 2002.

10. Santos VLCG, Sawaia BB. The pouch acting as a mediator between "being a person with an ostomy! And "being a professional": analysis of a pedagogical strategy. J Wound Ostomy Continence Nurs. 2001;28(4):206-14.

11. Turnbull GB, Erwin-Toth P. Ostomy care: foundation for teaching and practice. Ostomy Wound Manage. 1999;45(1A Suppl):23S-30S.

12. Paula RAB, Santos VLCG. Estudo retrospectivo sobre as complicações do estoma e da pele periestoma em ostomizados da cidade de São Paulo. Rev Esc Enferm USP. 1999;33(n.esp):63-73.
13. Robertson I, Leung E, Hughes D, Spiers M, Donnelly L, Mackenzie I, et al. Prospective analysis of stomarelated complications. Colorectal Dis. 2005;7(3):279-85.

14. Cesaretti IUR, Borges LLN, Greco APC. A tecnologia no duidar de ostomizados: a questão dos dispositivos. In: Santos VLCG, Cesaretti IUR. Assistência em estomaterapia: cuidando do ostomizado. São Paulo: Atheneu; 2000. p. 173-93.

15. White $M$, Berg K. A new flangeless adhesive coupling system for colostomy and ileostomy. Br J Nurs. 2005; 14(6):325-8.

16. Berg K, Seidler H. Randomized crossover comparison of adhesively coupled colostomy pouching systems. Ostomy Wound Manage. 2005;51(3):30-2.

17. Davenport R, Sica J. A new modern drainable appliance for people with ileostomies. Br J Nurs. 2003;12(9):571-5.

18. Centellas Morral M. Evaluación clínica de un nuevo dispositivo para personas colostomizadas. Rev Rol Enferm. 2000; 23(4):293-300.

19. Nielsen LF, Blume $N$, Romme $T$, Samuelsen $P$, Everland $H$, Ifversen $P$, et al. Skin changes induced by a zinc oxide dressing compared with a hydrocolloid dressing in healthy individuals. Skin Res Technol. 2005;11(2):140-51.

20. Burch J, Sica J. Colostomy products: an update on recent developments. Br J Community Nurs. 2004;9(9):373-8.

\section{Agradecimentos}

Agradecemos às colegas enfermeiras, pós-graduadas em estomaterapia Adriana Carla Lessa Pereira Vasconcellos, Angélica dos Santos Boness e Elaine Cristina Canova Barros pela colaboração durante a coleta de dados e elaboração do primeiro relatório. 